\title{
Protección SOCiAL CON ENFOQUe DE DERECHOS PARA LA AMÉRICA LATINA DEL SIGLO XXI
}

Simone Cecchini*

\section{Resumen}

Desde comienzos del siglo xxi, las políticas de protección social en América Latina han tenido un importante desarrollo, que ha resultado en la ampliación de la inversión pública social y la cobertura poblacional de las prestaciones. Muchas de las reformas de las políticas y la formulación de nuevos programas se relacionan de manera creciente con una visión que promueve la realización de los derechos económicos y sociales de todos los ciudadanos y las ciudadanas, y que avanza la agenda de la igualdad que se está instalando en la región. En este artículo se argumenta a favor de la adopción de un enfoque de derechos en la protección social, tanto por su valor ético y normativo como por su valor instrumental, especialmente en relación con la superación de la pobreza y el mejoramiento del bienestar de toda la población. Se hace en particular un llamado a seguir construyendo el Estado de bienestar, no obstante el complejo escenario económico actual.

Palabras clave: política social, protección social, enfoque de derechos, inversión social, desarrollo, América Latina.

\section{RIGHTS-BASED SOCIAL PROTECTION FOR 21ST CENTURY LATIN AMERICA}

\begin{abstract}
Since the beginning of the 21st Century, the significant progress of social protection policies in Latin America has resulted in higher social public investment and broader population coverage. Many of the policy reforms and the formulation of new program-
\end{abstract}

* Magíster en economía internacional. Oficial de Asuntos Sociales de la División de Desarrollo Social, Comisión Económica para América Latina y el Caribe (CEPAL), Santiago de Chile (Chile). simone.cecchini@cepal.org

Recibido: 1 de febrero de 2016 / Modificado: 20 de abril de 2016 / Aceptado: 3 de mayo de 2016

Para citar este artículo

Checchini, S. (2016). Protección social con enfoque de derechos para la América Latina del siglo XXI. OPERA, 18, pp. 11-33. DOI: http://dx.doi.org/10.18601/16578651.n18.03 
mes relate in a growing manner to a vision promoting the realization of the economic and social rights of all citizens, which fosters the equality agenda in the region. This article argues in favour of the adoption of the rightsbased approach to social protection, both for its ethical and normative value as well as for its instrumental value, especially with respect to overcoming poverty and improving the population's welfare. In particular, it calls for the continuation of efforts to build the welfare state, notwithstanding the current complex economic scenario.

Key words: Social policy, social protection, rights-based focus, social investment, development, Latin America.

\section{INTRODUCCIÓN}

Después de una década caracterizada por el crecimiento económico y la reducción de la pobreza y la desigualdad, América Latina se enfrenta actualmente a un escenario complejo, de bajo crecimiento y escasa o nula reducción de la pobreza. La CEPAL (2015a) ha estimado un retroceso de $-0,4 \%$ del PIB regional en 2015, año en que dos grandes economías, Brasil y Venezuela, se encuentran en fuerte recesión. Para 2016, se prevé una contracción de $-0,6 \%$, debido a un entorno global difícil en el cual se mantiene el bajo crecimiento de los países desarrollados, una importante desaceleración en las economías emergentes, en particular China; una creciente volatilidad en los mercados financieros y bajos precios de las materias primas, de las cuales dependen muchas de las economías de la región. A su vez, entre 2012 y 2014, la incidencia de la pobreza se ha mantenido estable en un $28 \%$ de la población, y la indigencia ha aumentado de 11,3 a 12,0\% (CEPAL, 2015b). A los problemas de carácter económico y social, se suma además un momento de grandes dificultades para las coaliciones políticas progresistas que en muchos casos lideraron los avances a lo largo de la última década, siendo el caso de Brasil ilustrativo.

Estas dificultades ponen a las políticas públicas en los países de la región -y en particular la política social- en una encrucijada. La disminución de los recursos fiscales lleva típicamente a ajustes y pugnas entre las múltiples demandas de financiamiento y los escasos recursos a disposición de los países. Frecuentemente, estos ajustes se orientan de manera casi automática a recortar la inversión social más que otros tipos de gastos, dejando así en segundo plano la importancia de seguir fortaleciendo el Estado de bienestar.

El Estado tiene un rol decisivo en la provisión de bienestar y, según señala SeguraUbiergo (2012, p. 16), su objetivo debe ser "asegurar un mínimo de bienestar a todos sus ciudadanos - protegiéndolos frente a los riesgos de desempleo, enfermedad, vejez y maternidad-y proveer una acumulación adecuada de capital humano a través de la inversión en salud y educación”. Por supuesto, el Estado no es el único actor, dado que el mercado, las familias y las comunidades también producen y reproducen riqueza, seguridad y oportunidades (Esping-Andersen, 1990 y 1999). Sin embargo, el Estado de bienestar desempeña algunas funciones muy claras: i) desmercantiliza el acceso de la población al bienestar 
mediante la provisión de bienes, servicios y transferencias que no dependen de la posición que la persona ocupa en el mercado laboral, ni de su capacidad de compra en el mercado; ii) desfamiliariza el acceso al bienestar al otorgar beneficios, servicios y transferencias que no dependen de la pertenencia a una familia; iii) regula y afecta ciertos aspectos del comportamiento de los agentes de mercado y de los integrantes familiares, modificando así el orden "espontáneo" en estas esferas; y iv) redistribuye recursos, pues al recaudarlos y distribuirlos en la forma de bienes, servicios y transferencias, no ajusta las prestaciones en forma exacta al aporte individual, sino que opera en diversas modalidades de solidaridad, ancladas en fuentes de financiamiento y criterios de elegibilidad (Esping-Andersen, 1990, 1999 y 2002).

En América Latina, la experiencia de la crisis de la deuda de los años ochenta enseńa que los fuertes ajustes a la inversión pública social llevan a graves consecuencias en términos de bienestar. La pobreza aumentó de 40,5\% en 1980 a $48,4 \%$ en 1990 , y solamente 25 años más tarde la región volvió a los mismos niveles de pobreza precrisis; a su vez, el pIB per cápita disminuyó significativamente y regresó al nivel de 198014 años después (CEPAL, 2010) (figura 1). Este desfase temporal indica que si bien la crisis económica fue muy fuerte, el impacto social fue aún peor, debido a los excesivos recortes a la inversión social, las privatizaciones de los seguros de salud y las pensiones, y la implementación de insuficientes y desarticulados programas sociales de emergencia.

En este artículo se argumenta que invertir en lo social significa no solo paliar los efectos de las crisis en las condiciones de vida de las personas, sino promover el bienestar y el desarrollo económico. La inversión social aumenta el bienestar: educar significa tener ciudadanos informados, con mejores capacidades para emprender y mejores condiciones de acceso a empleos productivos y de calidad, y de participación en diversos ámbitos de la vida política y social. Un sistema de salud eficaz permite una vida más larga y de mejor calidad, al tiempo que las prestaciones por desempleo y el salario mínimo reducen la pobreza (CEPAL, 2006; Cecchini y Martínez, 2011; Boyer, 2015). Por el contrario, la falta de inversión social acarrea costos y pérdidas de ingresos, como se ha evidenciado en los estudios sobre el costo del hambre (Martínez y Fernández, 2007 y 2009). Es así como, "el desarrollo social inclusivo y la mejora de las condiciones de vida de la población son un requisito necesario para asegurar la prosperidad económica" (CEPAL, 2015c, p. 9). Los recursos bien invertidos en lo social-además de contribuir a realizar los derechos económicos, sociales y culturales de toda la población- fortalecen las capacidades humanas a lo largo de todo el ciclo de vida y acarrean importantes ganancias en términos de productividad.

Para el desarrollo de América Latina en el siglo XXI, en particular, cobra aún mayor relevancia que en el pasado invertir en capacidades humanas, ya que las economías se basan siempre más en generar activos intangibles -ideas, destrezas y redes- que en los estímulos a la inversión en maquinarias y activos físicos orientados a la producción de bienes tangibles (Evans, 2008). 
FIGURA 1. AMÉRICA LATINA: PIB PER CÁPITA Y POBREZA, 1980-2013 (1980=100)

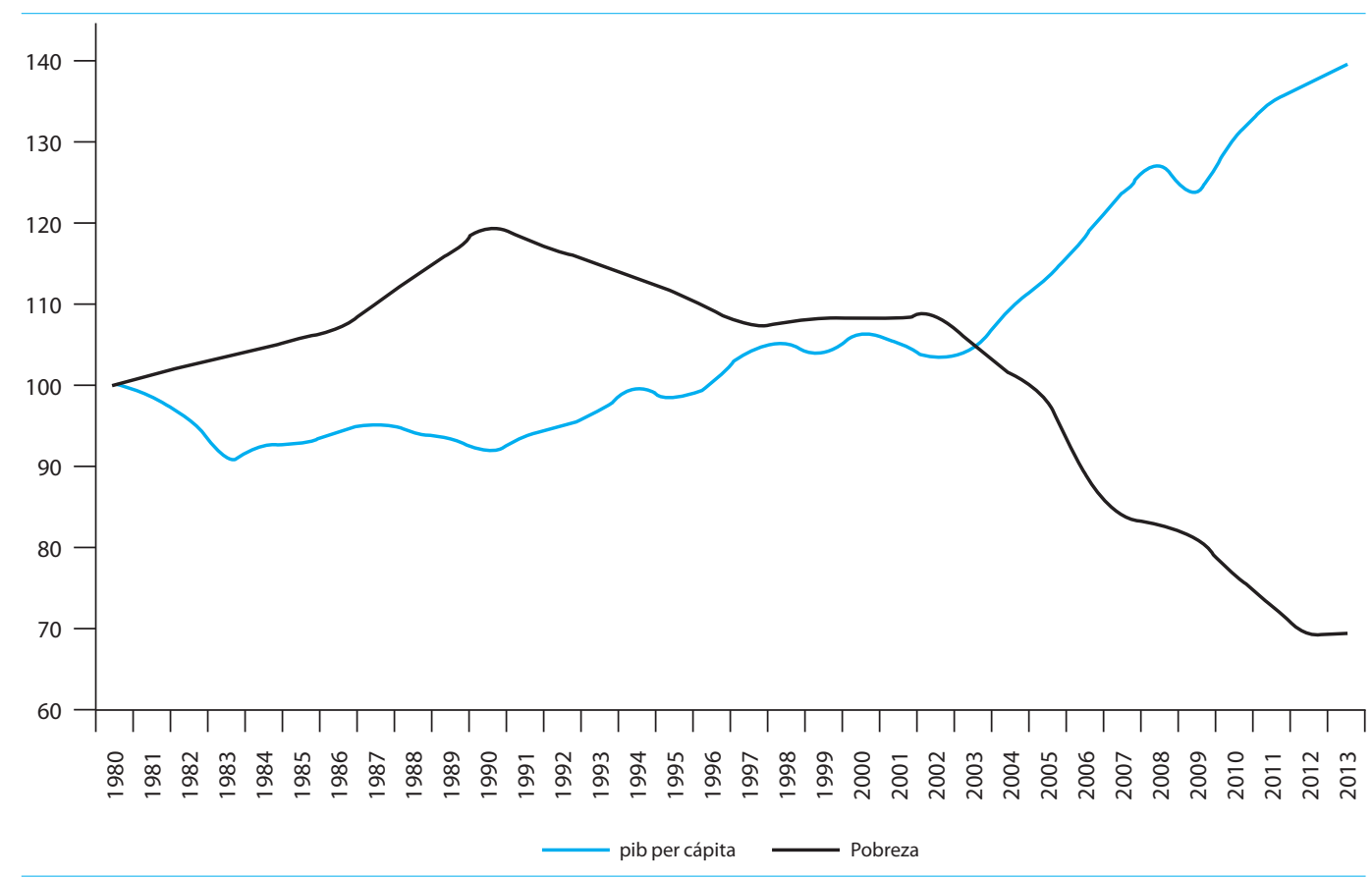

Fuente: elaboración propia, sobre la base de cepalstat.

En la región, además de seguir reforzando los niveles de inversión social, es necesario implementar políticas sociales con enfoque de derechos. Los elementos centrales del enfoque de derechos en el diseño, la implementación, el monitoreo y la evaluación de las políticas públicas -la igualdad y no discriminación, integralidad, institucionalidad, participación ciudadana, transparencia y rendición de cuentas (Cecchini y Rico, 2015; Sepúlveda, 2014) - no solo tienen un valor ético, sino que son fundamentales para que la inversión social obtenga los resultados deseados en términos de bienestar. Si bien en la región queda mucho por avanzar, el enfoque de derechos es clave para sustentar la necesaria articulación entre el Estado y la sociedad civil y así fomentar políticas sociales más efectivas.

Para esto, se requiere de una acción decidida del Estado, porque el logro de impactos mediante la inversión en capacidades humanas -educación y capacitación, salud, nutrición, entre otras- depende de la calidad de las políticas públicas. Pero también, para obtener los resultados deseados es necesario contar con el compromiso activo de la población, porque la pasividad frente a la prestación de servicios sociales por parte de los individuos, las familias y las comunidades podría llevar a resultados subóptimos o hasta contraproducentes (Evans, 2008). 
Este artículo se centra en un ámbito clave de la política social -la protección social ${ }^{1}-$, y argumenta a favor de la adopción de un enfoque de derechos en ella, tanto por su valor ético y normativo como por su valor instrumental-especialmente en relación con la superación de la pobreza y la reducción de las desigualdades-. Para estos fines, se destacan los avances en la ampliación de la cobertura de la protección social a lo largo de la última década y las grandes brechas que aún quedan por cerrar, y se propone que la región avance hacia políticas de protección social de corte universal con enfoque de derechos, destacando qué significa esto en concreto para la política pública y cuáles son sus alcances y desafíos. Finalmente, se concluye analizando algunos de los desafíos más importantes que se deben enfrentar para lograr avanzar hacia una política de protección social con enfoque de derechos y para construir un Estado de bienestar.

\section{COBERTURA DE PROTECCIÓN SOCIAL: PROGRESOS Y BRECHAS}

Según el enfoque de derechos, las políticas públicas deben caracterizarse por la progresividad y la no regresividad. Progresividad significa reconocer que los derechos económicos y sociales forman parte de un proceso, en tanto requieren el incremento y mejoramiento de los recursos humanos, físicos, institu- cionales y financieros para transitar de una titularidad formal (de jure) a una titularidad efectiva (de facto). A su vez, no regresividad significa que no se deben adoptar políticas y normas jurídicas que empeoren la situación de los derechos económicos y sociales de los que goza la población (Cecchini y Rico, 2015).

En lo que concierne a la evolución de la protección social, a partir de comienzos del siglo xxi los países de América Latina -en distintos momentos, grados y formas que dependen de su nivel de desarrollo socioeconómico y de su institucionalidad-han logrado expandir progresivamente la cobertura de la protección social, tanto en términos de cobertura poblacional como de mejoramiento de las prestaciones. Los avances se caracterizan, entre otros aspectos, por la implementación de programas integrales para la reducción de la pobreza -asociados al fortalecimiento de los activos y las capacidades humanas-, la construcción de pilares no contributivos de pensiones, la ampliación del acceso a la salud y la formalización de los trabajadores.

Para continuar en la senda del desarrollo en el actual sombrío escenario económico, es necesario seguir progresando y no retroceder en la cobertura de la protección social. Si bien los avances en protección social han sido importantes, aún hay grandes desafíos pendientes, ya que el acceso a las prestaciones de protección social vía mercado laboral sigue

1 Como destacan Cecchini y Martínez (2011), protección social no es sinónimo de política social, sino que es uno de sus componentes, junto con las políticas sectoriales - por ejemplo salud y educación-y la promoción social. A su vez, los componentes de la protección social son el contributivo (seguridad social), no contributivo (asistencia social) y la regulación del mercado laboral. 
siendo una promesa largamente incumplida (CEPAL, 2006; Cecchini y Martínez, 2011; Lo Vuolo, 2009).

En el año 2013, en América Latina 165 millones de personas vivían en la pobreza -el $28 \%$ de los habitantes de la región-, de las cuales 69 millones -el $12 \%$ - se encontraban en la pobreza extrema. Si a la población que vive en la indigencia o la pobreza se suma aquella que continúa viviendo en una situación de vulnerabilidad, resulta que el 51\% de los latinoamericanos no tienen ingresos suficientes para enfrentar una eventual crisis económica (CEPAL, 2015c) ${ }^{2}$.

La difícil situación social de la población latinoamericana tiene una estrecha relación con lo que ocurre en el mercado laboral. Este en promedio es responsable de un $80 \%$ de los ingresos familiares. Sin embargo, no siempre logra convertirse en el motor para que las personas y los hogares superen la pobreza y accedan al bienestar, y, al contrario -por su elevada segmentación y altos niveles de informalidad-, termina contribuyendo a producir y exacerbar las desigualdades.

Al 2013, el $51 \%$ de la población ocupada se insertaba en sectores de productividad media-alta, integrándose al mercado formal del trabajo y contando con algún tipo de protección social ante contingencias y riesgos estructurales. Sin embargo, el resto de la población ( $49 \%$ ) continuaba excluida del mercado formal del trabajo y, por ende, carecía de acceso a tales mecanismos. Preocupa, en particular, la mayor proporción de mujeres ocupadas en sectores de baja productividad $(53 \%)$ en comparación con los hombres (47\%). Asimismo, cerca del $45 \%$ de los asalariados no contaban con un contrato formal de trabajo en 2013, cifra que da cuenta de capas medias que quedan excluidas de un tipo de relación laboral contractual y, en consecuencia, se vuelven más vulnerables.

No obstante la existencia de estas dualidades y exclusiones, hay que reconocer que hace una década la situación era peor y que la región ha logrado avances gracias a la combinación virtuosa de crecimiento económico con creación de empleo y políticas públicas orientadas al mejoramiento del bienestar de la población. Los avances han sido generalizados, si bien los niveles de inversión y cobertura son muy distintos según los diferentes regímenes de bienestar que caracterizan a los países de la región.

En esta sección se muestra la evolución de algunos indicadores de esfuerzo y cobertura de protección social entre los años 2000 y

2 La CEPAL (2010) ha definido la situación de vulnerabilidad a partir de los ingresos expresados en grandes cortes de líneas de pobreza, ordenando a la población en cuatro grandes categorías: i) indigentes o altamente vulnerables a la indigencia, que incluye a quienes se encuentran en situación de indigencia o en sus límites y que, por tanto, tienden a entrar y salir de dicha situación (hasta 0,6 líneas de pobreza); ii) pobres o altamente vulnerables a la pobreza, es decir, aquellos cuyos ingresos son inferiores o cercanos a la línea de pobreza y que entran y salen de esa condición en ciclos económicos normales (entre 0,6 y 1,2 líneas de pobreza); iii) vulnerables a la pobreza, que comprenden a aquellos cuyos ingresos se sitúan entre 1,2 y 1,8 líneas de pobreza, y iv) no vulnerables, que incluye a aquellas personas cuyos ingresos son superiores a 1,8 líneas de pobreza. 
2012, no solo para el promedio regional, sino también para tres grupos de países - de brechas modestas, moderadas y severas- clasificados según la tipología de regímenes de bienestar presentada en Cecchini, Filgueira y Robles $(2014)^{3}$ (tabla 1). Esta clasificación se inserta en la amplia literatura que ha caracterizado y tipificado los estados de bienestar en América Latina (véase por ejemplo Barba, 2013; Martínez Franzoni, 2008; Mesa-Lago, 1977).

\section{TABLA 1. AMÉRICA LATINA: GRUPOS DE PAÍSES SEGÚN BRECHAS DE BIENESTAR, ALREDEDOR DE 2012}

\begin{tabular}{|c|c|c|}
\hline Brechas severas & $\begin{array}{c}\text { Brechas } \\
\text { moderadas }\end{array}$ & $\begin{array}{l}\text { Brechas } \\
\text { modestas }\end{array}$ \\
\hline $\begin{array}{l}\text { Bolivia, El Salva- } \\
\text { dor, Guatema- } \\
\text { la, Honduras, } \\
\text { Nicaragua y } \\
\text { Paraguay }\end{array}$ & $\begin{array}{l}\text { Colombia, Ecua- } \\
\text { dor, México, } \\
\text { Perú y República } \\
\text { Dominicana }\end{array}$ & $\begin{array}{l}\text { Argentina, Bra- } \\
\text { sil, Chile, Costa } \\
\text { Rica, Panamá, } \\
\text { Uruguay y Vene- } \\
\text { zuela }\end{array}$ \\
\hline
\end{tabular}

Fuente: Cecchini, Filgueira y Robles (2014).

Los países de brechas severas, moderadas y modestas no se diferencian solamente por los niveles de PIB per cápita sino también por su demografía y las características de sus mercados laborales. Los países con brechas severas de bienestar tienen una estructura demográfica que responde a relativamente elevados niveles de fecundidad, con una población joven. Su débil mercado de trabajo se caracteriza por escasos empleos formales, bajos salarios o remuneraciones y una carga de dependientes no solo conformados por jóvenes, sino por un alto contingente de trabajadores familiares no remunerados y del sector informal de subsistencia. A su vez, los países con brechas moderadas de bienestar tienen una estructura demográfica que responde a descensos en la fecundidad de la década de los setenta en adelante y, como consecuencia, tienen una menor proporción de jóvenes entre sus dependientes; experimentan un mercado de trabajo más formalizado que el grupo anterior, pero en algunos casos enfrentan un envejecimiento incipiente de su población. Finalmente, los países con brechas modestas de bienestar son los que tienen mayor potencial para desarrollar un Estado de bienestar. Se caracterizan por fuertes descensos de la fecundidad durante o antes de la década de los sesenta, y una estructura más envejecida de su población. Si bien ostentan los niveles de formalización laboral más elevados en la región, sus sistemas de protección social continúan segmentados por la capacidad contributiva de los afiliados, reproduciendo las desigualdades que se generan en el mercado de trabajo (Cecchini, Filgueira y Robles, 2014).

3 Esta tipología se basa en distintos factores, entre los cuales destacan la capacidad de las sociedades de generar ingresos suficientes a través del mercado laboral y las capacidades de los Estados para proporcionar sustento y protección a quienes carecen de ingresos o acceden a ingresos insuficientes. Las variables utilizadas para realizar la clasificación fueron: PIB per cápita (en dólares del 2005), tasa de dependencia, inversión pública social por habitante, inversión pública social en seguridad y asistencia social (en porcentajes del PIB), asalariados que aportan al sistema de pensiones, asalariados que aportan al sistema de salud y el porcentaje de la población de 15 años y más que se encuentra ocupada y que vive bajo la línea de pobreza (Cecchini, Filgueira y Robles, 2014). 
Una forma de examinar el desempeńo de la protección social en los países de la región consiste en analizar el esfuerzo -en términos tributarios y de inversión pública social- junto con los logros en materia de cobertura. Como se aprecia en la tabla 2, en América Latina subsisten brechas evidentes en la provisión de bienestar: en promedio, solamente el $38 \%$ de la población ocupada tiene cobertura en pensiones y $52 \%$ en salud, mientras que en los países de brechas severas de bienestar esas proporciones apenas llegan a 18 y $25 \%$, respectivamente. Incluso en el caso de los países de brechas modestas de bienestar, la cobertura de la seguridad social no es universal, de modo que urge la búsqueda de alternativas para mejorar las respuestas estatales en este ámbito.

TABLA 2. AMÉRICA LATINA: INDICADORES DE ESFUERZO Y DE COBERTURA DE PROTECCIÓN SOCIAL, SEGÚN BRECHAS DE BIENESTAR, 2000-2012 ${ }^{A}$

\begin{tabular}{|c|c|c|c|c|c|c|}
\hline \multirow{2}{*}{ Indicador } & \multicolumn{3}{|c|}{ Brechas severas } & \multicolumn{3}{|c|}{ Brechas moderadas } \\
\hline & 2000 & 2012 & Variación (\%) & 2000 & 2012 & Variación (\%) \\
\hline \multicolumn{7}{|c|}{ Indicadores de esfuerzo } \\
\hline Ingresos tributarios (en \% del pib) & 13,7 & 16,9 & 23,4 & 13,6 & 15,9 & 16,9 \\
\hline Inversión pública social (en \% del pib) & 8,9 & 10,8 & 21,3 & 7,8 & 9,7 & 24,4 \\
\hline Inversión pública social por habitante (en dólares de 2005) & 117 & 218 & 86,3 & 304 & 490 & 61,2 \\
\hline \multicolumn{7}{|c|}{ Indicadores de cobertura } \\
\hline $\begin{array}{l}\text { Cobertura de pensiones ( } \% \text { de la población de } 65 \text { años } \\
\text { y más) }\end{array}$ & 14,7 & 17,7 & 20,4 & 20,3 & 25,0 & 23,2 \\
\hline Ocupados con cobertura de pensiones (\%) & 17,9 & 19,4 & 8,4 & 22,4 & 35,6 & 58,9 \\
\hline Ocupados con cobertura de salud (\%) & 20,7 & 25,3 & 22,2 & 30,5 & 65,6 & 115,1 \\
\hline \multirow[t]{2}{*}{ Indicador } & \multicolumn{3}{|c|}{ Brechas modestas } & \multicolumn{3}{|c|}{ América Latina } \\
\hline & 2000 & 2012 & Variación (\%) & 2000 & 2012 & Variación (\%) \\
\hline \multicolumn{7}{|l|}{ Indicadores de esfuerzo } \\
\hline Ingresos tributarios (en \% del pib) & 19,6 & 24,1 & 23,0 & 16,0 & 19,4 & 21,3 \\
\hline Inversión pública social (en \% del pib) & 17,7 & 22,2 & 25,4 & 12,2 & 14,8 & 21,3 \\
\hline Inversión pública social por habitante (en dólares de 2005) & 882 & 1558 & 76,6 & 487,0 & 818,8 & 68,1 \\
\hline \multicolumn{7}{|c|}{ Indicadores de cobertura } \\
\hline $\begin{array}{l}\text { Cobertura de pensiones (\% de la población de } 65 \text { años } \\
\text { y más) }\end{array}$ & 71,5 & 75,7 & 5,9 & 37,0 & 38,6 & 4,3 \\
\hline Ocupados con cobertura de pensiones (\%) & 58,1 & 67,1 & 15,5 & 29,1 & 37,8 & 29,9 \\
\hline Ocupados con cobertura de salud (\%) & 79,1 & 84,8 & 7,2 & 40,9 & 52,9 & 29,3 \\
\hline
\end{tabular}

a Promedios simples.

Fuente: elaboración propia, sobre la base de Cecchini, Filgueira y Robles (2014). 
Las tendencias entre 2000 y 2012 de los tres indicadores de esfuerzos presentados en la tabla 2 son positivas para los tres grupos de países: los ingresos tributarios y la inversión pública social, tanto como proporción del PIB como en términos absolutos, aumentaron. Los ingresos tributarios y la inversión social en términos de porcentajes del piB crecieron un $21,3 \%$, mientras que -gracias al crecimiento del PIB- el aumento real de la inversión social en dólares por habitante fue muy superior $(68,1 \%)$.

En relación con los indicadores de cobertura, se puede apreciar que las diferencias en cobertura de pensiones y salud son muy grandes entre los países de brechas modestas, moderadas y severas. Alrededor del año 2000 , las diferencias entre el grupo de brechas modestas y el de brechas severas eran de 58 puntos porcentuales en cobertura de pensiones entre la población mayor de 65 años, y 48 y 60 puntos porcentuales en afiliación de los ocupados a sistemas de pensiones y salud, respectivamente. Dichas diferencias se mantuvieron -o hasta aumentaron- a lo largo de la década porque los avances fueron más decididos en los países de brechas modestas de bienestar. En estos últimos, entre los años 2000 y 2012, la cobertura de pensiones entre la población mayor de 65 años se incrementó del 71,5 a 75,7\%, y los ocupados afiliados a un sistema de pensiones pasaron de 58,1 a $67,1 \%$. En materia de cobertura de salud, en el año 2000 el 79,1 \% de los ocupados de los países de brechas modestas estaban cubiertos por algún tipo de seguro de salud, mientras que dicho indicador no superaba el 30,5 y el 20,7\% entre los países de brechas moderadas y severas, respectivamente. Al 2012, el progreso más significativo en materia de seguros de salud entre los ocupados fue entre los países de brechas moderadas, quienes lograron doblar la cobertura. Este avance se sumó a importantes logros en materia de pensiones, lo que contribuye a aumentar la diferencia con los países de brechas severas pero sin disminuir la distancia respecto a los países con brechas modestas de bienestar (Cecchini y Vargas, 2015).

Además de los avances en los indicadores presentados en la tabla 3 , cabe destacar otros dos ámbitos en los cuales la región ha progresado sustancialmente: las transferencias monetarias a familias con hijos-que, por ejemplo, bajo la forma de programas de transferencias condicionadas alcanzaban al 2013 el 21,5\% de los latinoamericanos, comparado con el 5,7\% en 2000 (CEPAL, 2015c)-y la protección al trabajador, en la forma de seguros de enfermedad y de desempleo, sumado a políticas de derechos laborales, tales como indemnización, horas extras o licencias. Desde una perspectiva integral, asimismo, se destacan esfuerzos sinérgicos impulsados por algunos países de la región (como Brasil, Chile, Colombia y Uruguay) para conformar verdaderos sistemas o subsistemas de protección social dirigidos a la primera infancia, donde se incorpora también el componente de cuidado (Cecchini, Filgueira y Robles, 2014). 


\section{EL ENFOQUE DE DERECHOS EN LA POLÍTICA SOCIAL Y LA PROTECCIÓN SOCIAL ${ }^{4}$}

Lograr la plena titularidad de los derechos económicos y sociales de todas las ciudadanas y los ciudadanos significa que estos están incluidos en la dinámica del desarrollo y gozan del bienestar, lo que implica una efectiva pertenencia a la sociedad ("ciudadanía social") (Marshall, 1950) . También supone un freno -mediante la acción deliberada del Estado-a las desigualdades socioeconómicas que privan a muchos de una real pertenencia a la sociedad, así como un reconocimiento de todos los miembros de la sociedad sin distinciones de género, raza, etnia, edad, pertenencia a grupos socioeconómicos específicos o localización geográfica (CEPAL, 2006). En la medida en que los derechos económicos y sociales se materializan como derechos inalienables de ciudadanía, es posible avanzar hacia una mayor igualdad en el acceso al bienestar y dar un fundamento ético a las políticas sociales (Hopenhayn, 2001).

Según lo planteado por la cepal (2006), el enfoque de derechos nace de la idea normativa de la sociedad de ciudadanos y de la igualdad. De hecho, Bobbio (1996) argumenta que la lógica detrás de los derechos humanos a la educación, la salud o el trabajo es eminentemente igualitaria, ya que los derechos económicos y sociales buscan reducir las desigualdades entre ricos y pobres, y poner a un creciente número de personas en mejores condiciones en relación con los más aventajados. La plena titularidad de derechos es, de este modo, el fundamento ético para avanzar por el camino de la igualdad, en la medida en que requiere de un pacto fiscal y de un Estado con vocación redistributiva a través de políticas universales y umbrales progresivos en las prestaciones. En este marco, estar socialmente protegido es consecuencia de un derecho básico de pertenencia a la sociedad, es decir, de participación e inclusión (CEPAL, 2006).

El enfoque de derechos contribuye a promover la ciudadanía social: todos son titulares de derechos considerados inalienables, incluidos los derechos sociales y económicos que hacen a la protección social. La plena ciudadanía, a su vez, tiene una relación muy fuerte con la cohesión social y el sentido de pertenencia, y constituye el fundamento de la vida democrática. Como se observa en los países que cuentan con Estados de bienestar desarrollados-como en los países nórdicos en Europa-, la dialéctica virtuosa entre la construcción de pactos sociales entre actores diversos, el manejo negociado de los conflictos, la expansión de derechos sociales y el sentido de pertenencia constituye la gran palanca de la cohesión social.

El enfoque de derechos en la protección social implica que las políticas públicas deben tener por objeto facilitar un mayor disfrute

\footnotetext{
$4 \quad$ Esta y la siguiente sección se basan en Cecchini y Rico (2015).

5 En este sentido, la falta de titularidad de los derechos económicos y sociales remite a un concepto de pobreza que no se refiere solo a la condición socioeconómica, sino también a una privación de ciudadanía (CEPAL, 2007).
} 
de los derechos económicos y sociales de toda la población, basándose en las normas y los principios de derechos humanos consagrados en constituciones, leyes y tratados internacionales. Vivir protegidos no es solo una conquista social o un logro de los gobiernos; es exigible como imperativo de la ciudadanía (Cepal, 2006).

Por supuesto la protección como garantía ciudadana no es el único enfoque existente en la región y en el mundo. Hacia fines de los ańos noventa, el Banco Mundial desarrolló el marco de análisis del manejo social del riesgo para desarrollar políticas de protección social. Este marco define una serie de estrategias y mecanismos que -ante la ausencia o deficiencia de los mercados- pueden implementarse para identificar tempranamente las diversas fuentes y tipos de riesgos, prevenirlos, mitigarlos y superarlos (Holzmann y Jorgensen, 1999; Serrano y Raczynski, 2003). Esos mecanismos se conciben como intervenciones acotadas en el tiempo. Básicamente, el manejo social del riesgo intenta destacar el impacto de las imperfecciones del mercado en el riesgo que enfrentan las familias (Munro, 2008) y la necesidad de generar medidas públicas de protección social para reducir la vulnerabilidad, activar el consumo y promover la equidad en la gestión de los riesgos de los individuos, hogares y comunidades, especialmente de los más pobres y vulnerables. En años recientes, este organismo ha complementado esta formulación con la necesidad de brindar una protección social eficaz a todos los ciudadanos. Las nuevas propuestas enfatizan los desafíos que impone la informalidad como barrera de entrada a la protección social y la fragmentación en el acceso a los beneficios de las políticas de protección social para trabajadores formales e informales, así como la necesidad de encontrar mecanismos para extender la protección social contributiva a todos los ciudadanos, independientemente de su situación laboral, complementando esta acción con el fortalecimiento sustentable de su capital humano y empleabilidad (Ferreira y Robalino 2010; Ribe, Robalino y Walker, 2010). Si bien en las nuevas propuestas del Banco Mundial se menciona la importancia de realizar los derechos económicos y sociales de la población, el énfasis está principalmente en el logro de una mayor eficiencia en el uso de los recursos.

Además de la existencia de enfoques alternativos, cabe también destacar que existen preocupaciones por la supuesta rigidez del enfoque de derechos, que podría acotar de manera poco conveniente la autonomía de quienes llevan adelante las políticas públicas. $\mathrm{Al}$ respecto, cabe mencionar que el enfoque de derechos no prescribe el contenido exacto de las políticas de protección social, ya que los Estados tienen la facultad de formular las políticas que sean más adecuadas a sus circunstancias (Abramovich, 2006; Sepúlveda, 2014; IPPDH, 2014). El enfoque de derechos no plantea una receta única aplicable de la misma manera en contextos diversos, ni intenta eludir o desplazar a la política; lo que hace es ofrecer una orientación general para el debate político y brindar un marco conceptual para la planificación de las políticas públicas. Las obligaciones que surgen de las constituciones y de los tratados de derechos humanos no le restan espacio a la interpretación y disputa 
política, sino que ponen de relieve las acciones prioritarias que el Estado tiene el deber de realizar (IPPDH, 2014).

Además del Estado, otros tres actores -el mercado, las familias y las organizaciones sociales y comunitarias- son también proveedores de bienestar y protección social. Sin embargo, ninguno de ellos puede jugar el papel central en la promoción de los derechos económicos y sociales que tiene el Estado. Si la protección social se dejara principalmente en manos del mercado, los más débiles quedarían excluidos, como queda demostrado con la falta de cobertura de trabajadores informales y pobres en los sistemas privados de pensiones. Si la protección social quedara primariamente en manos de las familias, las mujeres seguirían viéndose obligadas a dedicar gran parte de su tiempo a la función que han desempeñado históricamente: el trabajo no remunerado de cuidado de nińos, ancianos, enfermos y personas con discapacidad. Esto disminuiría sus posibilidades de participar en el trabajo remunerado, tener ingresos propios y, por tanto, ejercer su ciudadanía económica. Por último, las organizaciones sociales y comunitarias no cuentan con suficientes recursos ni con mecanismos para representar al conjunto de la ciudadanía para asumir la responsabilidad social completa en este ámbito (Cecchini y Martínez, 2011).

Más allá de adoptar un genérico "discurso de los derechos", es necesario definir de manera precisa el significado y las implicaciones del enfoque de derechos en la protección social. Para esto, siguiendo a Sepúlveda (2014, pp. 21-35), presentamos aquí los elementos esenciales de las políticas públicas y los pro- gramas de protección social basados en un enfoque de derechos. Estos elementos son: i) igualdad y no discriminación, ii) integralidad, iii) institucionalidad, iv) participación, v) transparencia y acceso a la información y vi) rendición de cuentas.

El principio de igualdad -asociado a la prohibición de la discriminación- es el elemento fundamental de un enfoque de derechos. Dar cumplimiento al principio de igualdad no se agota en la prohibición de actos discriminatorios por diferentes razones, sino que obliga a los Estados a tomar medidas para lograr la igualdad sustantiva. Estas medidas deben tener por objeto la transformación de las condiciones estructurales que determinan las desigualdades de índole socioeconómico, étnicas, raciales, de género, identidad sexual, discapacidad y demás (IPPDH, 2014). En el campo de la protección social, esto requiere extender progresivamente la cobertura a toda la población de un país, dando prioridad y atendiendo las necesidades particulares de las personas o los grupos más desaventajados y asegurando la igualdad entre hombres y mujeres. El Sistema Único de Salud (sus) del Brasil, que se basa en el enfoque de derechos definido en la Constitución de 1988, y asegura que el acceso a la salud sea integral, universal y libre de costos, es un buen ejemplo de medida que proporciona el acceso universal a la protección social, de conformidad con la obligación de buscar progresivamente el disfrute de los derechos económicos y sociales por parte de toda la población (Robles y Mirosevic, 2013).

$\mathrm{La}$ interdependencia e indivisibilidad hacen necesario un enfoque integral de las políticas públicas en general y de la protección 
social en particular. La fragmentación y la falta de coordinación entre diversos programas, actores y niveles de gobierno responsables de la aplicación de políticas de protección social aumentan la probabilidad de que dichas políticas sean ineficaces y de que se vulneren los derechos de sus destinatarios. Por tanto, para que las políticas y los programas de protección social sean efectivos y conformes a los derechos humanos, han de ser parte de estrategias nacionales que promuevan una visión multidimensional del bienestar y una efectiva coordinación entre actores, superando la lógica institucional sectorialista. El programa Chile Solidario y el plan Brasil Sem Miséria representan buenos ejemplos de estrategias integrales de asistencia social y reducción de la pobreza en la región.

Un aspecto central del enfoque de derechos en la protección social es que las políticas y los programas estén establecidos y definidos a través de un marco institucional y jurídico adecuado, que comprende la Constitución, las leyes, los reglamentos y los documentos de política pública. La existencia de dicho marco institucional y jurídico constituye la expresión formal de las intenciones de un Estado, asignándoles un carácter vinculante. Una institucionalidad apropiada es, por tanto, necesaria para garantizar que las políticas de protección social sean políticas de Estado que cuenten con un compromiso a largo plazo y con los recursos financieros y humanos adecuados para su ejecución. La institucionalidad es necesaria también para que la protección social sea vista como un derecho y no como un privilegio por parte de las ciudadanas y los ciudadanos, así como para promover el princi- pio de rendición de cuentas. La Ley Orgánica de Asistencia Social (LOAS) de Brasil de 1993, y la Ley General de Desarrollo Social de México de 2004, son buenos ejemplos de provisión de claros marcos legales e institucionales.

La participación es uno de los principios básicos de la democracia y es crucial para la garantía de los derechos: sin participación no hay democracia plena ni expansión de derechos (IPPDH, 2014). Para que las políticas y los programas de protección social contribuyan al proceso de transformación de las dinámicas de poder que exigen los derechos humanos, deben garantizar una participación efectiva y significativa de la población. A diferencia de algunos procesos que son puramente formales, simbólicos o llevados a cabo para dar una pátina de legitimidad a políticas predeterminadas, la participación basada en los derechos pretende ser transformadora, y promueve y exige la participación activa, libre, informada y genuina de los destinatarios en todas las etapas de la formulación, aplicación y evaluación de las políticas que les afectan. La participación corresponde a un derecho en sí mismo -el derecho a participar en los asuntos públicos- y es conveniente desde el punto de vista de la sostenibilidad de las políticas o los programas de protección social. Sin mecanismos de participación, las políticas y los programas corren el riesgo de ser objeto de manipulación política. Asimismo, si los encargados de formular políticas públicas y los administradores de los programas no reciben insumos de los destinatarios, la eficacia y sostenibilidad de las medidas se ven socavadas. Brasil es uno de los países de la región que más ha avanzado en materia de participación 
ciudadana en la protección social. Según la LOAS, las políticas de asistencia social deben fomentar la participación social. En consecuencia, se ha creado el Consejo Nacional de Asistencia Social (CNAS), que tiene incidencia a nivel nacional, estatal y municipal, y una composición paritaria entre los miembros que pertenecen a la sociedad civil y al Gobierno. El cNAS regula la implementación de los servicios sociales, vota el presupuesto de asistencia social y monitorea la inversión, entre otros deberes (Robles y Mirosevic, 2013).

El derecho a la información, que se funda en los derechos a la libertad de expresión y participación política, es vital para el funcionamiento y la preservación de los sistemas democráticos. En particular, el derecho a acceder a la información pública se sustenta en el principio de difusión de las acciones de gobierno, que exige informar a la ciudadanía sobre las medidas que se adoptan en el ejercicio de la representación política. De este modo, el Estado debe promover la producción de información relevante que dé cuenta de sus acciones, permitir el acceso a esta información por parte de la población y desplegar estrategias para su adecuada difusión (IPPDH, 2014). Desde un enfoque de derechos, es esencial que todas las políticas y programas públicos de protección social sean transparentes y prevean un adecuado acceso a la información, a fin de evitar la corrupción, el abuso, la mala gestión y la manipulación política. Transparencia significa que las ciudadanas y los ciudadanos deben ser capaces de entender el contenido de las políticas y el funcionamiento de los programas, por ejemplo, en relación con los criterios de elegibilidad y los mecanismos de selección de los destinatarios, las prestaciones, así como los resultados de evaluaciones y estudios de impacto. Asimismo, la información relativa a políticas y programas de protección social debe ser proporcionada de manera fácil de comprender y por canales accesibles física y culturalmente. También es necesario que los funcionarios estatales estén capacitados para responder de manera cabal a solicitudes de información. Por último, se debe garantizar que el acceso a la información pública no vaya en detrimento del derecho a la privacidad de la información. En la región, varios programas de transferencias condicionadas han incorporado mecanismos para mejorar el acceso a la información, tales como líneas telefónicas gratuitas para los destinatarios del Bono de Desarrollo Humano en Ecuador y de Prospera en México.

Otro de los elementos esenciales de un enfoque de derechos es habilitar a las personas para que puedan reclamar la garantía efectiva de su derecho a la protección social y hacer efectiva la responsabilidad de los funcionarios públicos en casos de errores, abusos o mala administración. Esto significa que deben establecerse normas, procedimientos y controles para limitar la discrecionalidad de funcionarios, empleados y políticos en el ejercicio de la función pública, así como mecanismos para investigar y sancionar faltas cometidas por los servidores públicos. Sin mecanismos de rendición de cuentas o de reparación, las políticas de protección social tendrán menos probabilidades de ser entendidas en términos de derechos, y serán vistas como instrumentos de clientelismo, sujetos a manipulación política (Sepúlveda, 2014). A su vez, la rendición de 
cuentas "puede contribuir a mejorar la institucionalidad y efectividad de las políticas y los servicios sociales, generando una gestión más abierta y receptiva a las demandas sociales, y promoviendo una mayor participación de la ciudadanía en las políticas sociales” (IPPDH, 2014, p. 71). Varios países de la región han establecido mecanismos que permiten a los ciudadanos controlar a los funcionarios públicos encargados de la ejecución de programas de transferencias condicionadas mediante la presentación de una queja o denuncia en caso de irregularidades o de abusos de poder. En México, por ejemplo, el sistema de atención ciudadana recibe y maneja las quejas de los ciudadanos y destinatarios de Prospera, y antes de Oportunidades (Hevia de la Jara y Gruenberg, 2010).

En varios países de América Latina se ha avanzado considerablemente en el reconocimiento de los derechos económicos y sociales a nivel constitucional. Sin embargo, este reconocimiento no debe entenderse como precondición para la puesta en marcha de políticas diseñadas con un enfoque de derechos ni de la expresión formal de garantías sociales (Cecchini y Martínez, 2011). En la tabla 3 se puede apreciar en qué países se ha adoptado un discurso y enfoque de derechos en las políticas de protección social, y dónde existen garantías sociales explícitas, todas ellas en materia de salud ${ }^{6}$.
TABLA 3. AMÉRICA LATINA: ENFOQUE DE DERECHOS EN LA PROTECCIÓN SOCIAL

\begin{tabular}{|c|c|c|c|}
\hline País & $\begin{array}{l}\text { Reconoci- } \\
\text { miento cons- } \\
\text { titucional } \\
\text { de derechos } \\
\text { sociales }\end{array}$ & $\begin{array}{l}\text { Enfoque } \\
\text { de dere- } \\
\text { chos en } \\
\text { protec- } \\
\text { ción social }\end{array}$ & $\begin{array}{c}\text { ¿Ejemplos } \\
\text { de expresión } \\
\text { formal de } \\
\text { garantías } \\
\text { explícitas? }\end{array}$ \\
\hline Argentina & Sí & Sí & Sí \\
\hline Bolivia & Sí & Sí & Sí \\
\hline Brasil & Sí & Sí & Sí \\
\hline Chile & & Sí & Sí \\
\hline Colombia & Sí & Sí & Sí \\
\hline Costa Rica & Sí & Sí & \\
\hline Cuba & Sí & Sí & \\
\hline Ecuador & Sí & Sí & \\
\hline El Salvador & Sí & Sí & \\
\hline Guatemala & & Sí & Sí \\
\hline Haití & Sí & & \\
\hline Honduras & Sí & & \\
\hline México & Sí & Sí & \\
\hline Nicaragua & Sí & & \\
\hline \multicolumn{4}{|l|}{ Panamá } \\
\hline Paraguay & Sí & Sí & \\
\hline Perú & Sí & Sí & Sí \\
\hline $\begin{array}{l}\text { República } \\
\text { Dominicana }\end{array}$ & Sí & Sí & \\
\hline Uruguay & Sí & Sí & Sí \\
\hline Venezuela & Sí & Sí & \\
\hline
\end{tabular}

Fuente: Cecchini y Rico (2015).

6 Siguiendo a Gacitúa-Marió, Norton y Georgieva (2009), por garantía social se entiende un mecanismo específico que un Gobierno instala para realizar un derecho y que implica obligaciones inmediatas. En particular, se garantiza el acceso a los servicios, su calidad, financiamiento, monitoreo y revisión continua, así como la disponibilidad de mecanismos de compensación. 
Adoptar un enfoque de derechos en la protección social tiene sentido tanto por su valor ético y normativo como por su valor instrumental. Sin embargo, debemos reconocer que este enfoque enfrenta retos importantes, entre los que se destacan los que derivan de la judicialización de los derechos económicos y sociales. Existen también tensiones de orden conceptual, en particular alrededor de las condicionalidades que están presentes en varios programas sociales de la región, así como en torno a la focalización de las medidas. Sin la intención de resolver tajantemente los debates conceptuales en curso, en lo que sigue se busca dar cuenta, de manera sucinta, de los retos y delinear posibles salidas para debates que a veces se encierran en posiciones irreconciliables y poco constructivas.

En muchos países de la región aún está arraigada la visión según la cual los derechos económicos y sociales constituyen meras declaraciones de intención que no son exigibles por la vía judicial ni administrativa. En otros, como Brasil o Colombia, la consagración constitucional de los derechos económicos y sociales se ha traducido en una creciente jurisprudencia por parte de los tribunales (Sepúlveda, 2014). Sin embargo, la judicialización de la protección social conlleva una serie de riesgos y dificultades, tanto en materia de igualdad -dado que la población más pobre y vulnerable tiene mayores restricciones de acceso a la solución judicial, tanto por razones económicas como culturales- como de sobrecarga para el aparato judicial y de definición de funciones y competencias entre poderes del Estado, ya que la responsabilidad del diseño y el financiamiento de las políticas de protección social recae en los poderes legislativos y ejecutivos, y no en el poder judicial (Cecchini y Martínez, 2011). Como indica Artigas (2005), la garantía de universalidad de las prestaciones asociadas a la realización de derechos no se obtiene necesariamente a través de la naturaleza individual de los casos en que intervienen mecanismos y fallos judiciales, sino que surge de pactos sociales y fiscales concretos que comprometan a la sociedad en su conjunto. Sin pactos sociales constituidos en torno a los derechos económicos y sociales, que permitan a las sociedades darse un horizonte de sentido y de orientación, y que ayuden a definir con mayor precisión cuál es el abanico de acceso a prestaciones y activos al que todo ciudadano puede aspirar y cuáles son los plazos que la sociedad fija para el logro de la plena titularidad (CEPAL, 2006), difícilmente se puede mantener un sistema basado en garantías.

El caso de Colombia es emblemático, especialmente en relación con la tutela del derecho a la salud. En 1992, la Corte Constitucional ordenó al Instituto de Seguros Sociales proporcionar tratamiento a una persona que vivía en la indigencia y había perdido la vista. Se estableció que el Congreso no había cumplido su deber de adoptar una ley que se ocupara de la situación de personas que viven en condición de extrema pobreza. Además, en 1995, la Corte ordenó que el sistema de seguridad social pagara los gastos de tratamiento de salud en una clínica especializada de Estados Unidos para una niña que padecía de leucemia y necesitaba un trasplante complejo, considerando que en Colombia no había un tratamiento disponible. Como consecuencia 
de estos fallos, la jurisprudencia relativa al derecho a la salud llevó a algunos desenlaces indeseados, entre ellos una explosión de demandas. Entre 1999 y 2009, los tribunales colombianos recibieron 775.102 tutelas relacionadas con el derecho a la salud (entre el 25 y el $40 \%$ de todas las acciones de tutela presentadas en el país), con efectos negativos sobre la equidad, la sostenibilidad financiera y la eficiencia del sistema. Para enfrentar esta situación, en 2008 la Corte Constitucional dictó la sentencia T-760, en la cual acumuló 22 tutelas que ilustraban las principales falencias del sistema de salud y dictó órdenes de carácter estructural al Gobierno colombiano para enfrentarlas (Sepúlveda, 2014). La sentencia de la Corte Constitucional de Colombia muestra así que los litigios también pueden desencadenar procesos estructurales más igualitarios (Sepúlveda, 2014; Uprimny y Durán, 2014). La exigibilidad de derechos por vía judicial o administrativa, ya sea a través del reclamo individual, de una cadena de reclamos particulares o de acciones colectivas, puede dar visibilidad a un problema público que aún no es tomado en cuenta por los gobiernos. Estas situaciones llaman la atención sobre la necesidad de establecer circuitos de información que vinculen los sistemas de reclamo judicial y administrativo con los procesos de definición del problema, diseño y evaluación de políticas, para que estos operen en un sentido de captación anticipada o "alertas tempranas" de problemas que aporte a un diagnóstico más preciso y contribuya a establecer prioridades y tomar decisiones (IPPDH, 2014) ${ }^{7}$.

Hay que dar cuenta también de los debates en curso sobre las condicionalidades y la focalización. Estos dos instrumentos, utilizados con frecuencia por los programas de protección social en América Latina, resultan problemáticos desde una perspectiva de derechos. Sobre las condicionalidades se ha advertido que los derechos humanos no dependen del cumplimiento de ninguna condición y son inherentes a la persona (Sepúlveda, 2014) y al ejercicio de la ciudadanía. Asimismo, una fijación demasiado estricta en las condicionalidades puede generar una distinción poco afortunada entre pobres "merecedores" o "no merecedores" de asistencia, lo que choca con el principio de universalidad y violaría derechos humanos básicos con relación con el aseguramiento de un nivel mínimo de vida. Un aspecto problemático que se suma al de las condicionalidades es el de las sanciones que se aplican en caso de incumplimiento, porque al castigar a las familias con suspensiones o exclusiones de las prestaciones, se corre el riesgo de dejar en condición de mayor vulnerabilidad justamente a los más pobres. Mientras más fuertes sean las sanciones, más alta será la tensión con los derechos humanos (Sepúlveda, 2014).

\footnotetext{
7 Por ejemplo, la existencia de largas listas de espera para acceder a jardines infantiles públicos en varios países de la región constituye un alerta acerca del déficit de este servicio y de la imposibilidad de resolverlo a través del mercado para los estratos sociales de menores ingresos (IPPDH, 2014).
} 
En la práctica, algunos países están usando las condicionalidades para reforzar derechos en educación y salud y para fomentar el trabajo intersectorial, sin adoptar medidas punitivas. En el caso del programa Bolsa Família de Brasil, por ejemplo, el propósito del monitoreo de las condicionalidades en educación y salud, a cargo de la Secretaría Nacional de Ingresos de la Ciudadanía (sENARC) del Ministerio de Desarrollo Social y Lucha contra el Hambre (MDs), no es punir a las familias, sino entender las razones del incumplimiento, ofreciéndoles el acompañamiento de trabajadores sociales y ayudándolas a que puedan acceder a la educación y la salud (González de la Rocha, 2010). En Bolsa Família, las transferencias monetarias solo pueden suprimirse si las familias quedan en condición de suspensión por más de 12 meses y si, durante ese periodo, han recibido la debida asistencia y monitoreo por parte de los servicios sociales. Según Soares (2012), entre 2006 y 2008, solo el 4,5\% de las familias que no cumplieron con las condicionalidades perdieron las prestaciones monetarias.

A su vez, el debate sobre focalización y universalismo de las políticas y los programas de protección social es de larga data, y desde el campo de los derechos se han establecido diversos argumentos en contra de la excesiva importancia de la focalización en los programas de protección social. En primer lugar, se argumenta que, si bien han sentado los fundamentos para una selección de destinatarios basada en criterios técnicos y no clientelistas, esto también se puede lograr a través de prestaciones universales, que reducen las oportu- nidades de corrupción y ayudan a eliminar el estigma asociado a la asistencia social. En segundo término, la sofisticación de los procedimientos de focalización hace que muchas veces los criterios de elección se vuelvan opacos, lo que desde la perspectiva de los destinatarios redunda en una incomprensión del funcionamiento del programa. Asimismo, se ha puesto énfasis en que en situaciones de pobreza generalizada, los costos administrativos y los problemas mencionados podrían exceder los beneficios que usualmente se atribuyen a la focalización. Argumentamos aquí que la focalización es un instrumento para reducir las desigualdades y optimizar la distribución de recursos, mientras que la universalización de la protección social es el objetivo último. Atender de manera diferenciada no contraviene necesariamente el principio de universalidad de los derechos. Por el contrario, puede potenciar su ejercicio y disminuir la desigualdad, tal como ocurre con las políticas de acción positiva frente a minorías étnicas, y a grupos de corte socioeconómico, cultural, etario o de género que se encuentran en situaciones de mayor vulnerabilidad (Hopenhayn, 2001), que pueden enmarcarse en un "universalismo sensible a las diferencias" (Habermas, 1998). Por ello, la focalización (o acción positiva) de los servicios de protección social hacia los más vulnerables puede ser un camino adecuado para avanzar de manera incremental hacia la universalización de los derechos económicos y sociales, en un proceso de realización progresiva de acuerdo con los niveles de desarrollo (Cecchini y Martínez, 2011). 


\section{CONCLUSIONES}

Como señala Hardy (2014), el haber pasado de políticas sociales para las necesidades básicas, a políticas con enfoque de derechos ha sido "el más decisivo cambio introducido en el enfoque de las políticas sociales de la región" (p. 154). Se argumenta aquí que este cambio debe ser sostenido y fortalecido para avanzar hacia la construcción de un Estado de bienestar y progresar hacia el verdadero desarrollo, aún si la región está enfrentándose a un periodo de dificultades económicas y cambios de gobiernos. La construcción del Estado de bienestar es eminentemente progresiva, puesto que no ocurre de un día para otro, sino que es el resultado de un largo proceso (Pierson y Leimgruber, 2010). Asimismo, en la experiencia de países que han alcanzado un régimen de bienestar socialdemócrata o universal, en las categorías de Esping-Andersen (1990), dicha construcción no ocurrió como resultado del crecimiento económico, sino que comenzó cuando los países aún se encontraban en vías de desarrollo. Este es el caso de Noruega, donde la época de expansión del Estado de bienestar fue entre 1945 y los ańos setenta (Sønneland, 2014), con base en la negociación colectiva y antes del descubrimiento de los pozos petrolíferos y el gran enriquecimiento del país.

Es justamente en periodos difíciles y de crisis que hay que seguir apostando al desarrollo, lo que requiere lograr pactos sociales y fiscales entre los distintos sectores sociales para la construcción de sistemas de protección social universales (CEPAL, 2014). Estos pactos deben determinar la estructura, el nivel impositivo y la asignación de las inversiones, considerando mayores esfuerzos públicos y privados, y un uso lo más eficiente posible de los recursos disponibles. En esta ecuación también hay que considerar que los costos económicos asociados a la falta de protección de la población no son irrelevantes y que, por tanto, no proteger supone un elevado costo.

Como destacan Balakrishnan, Elson, Heintz y Lusiani (2011), para realizar los derechos económicos y sociales de todas las ciudadanas y los ciudadanos, los países tienen la obligación de usar los máximos recursos disponibles, lo que implica tomar en cuenta no solo factores como la tributación (y la asistencia de la cooperación internacional en el caso de los países más pobres) y la inversión pública, sino también la política monetaria y el financiamiento de la deuda pública. También implica que "los recursos por considerar no son exclusivamente aquellos disponibles en las arcas estatales, sino que se deben incluir los recursos provenientes o que potencialmente podrían provenir del sector privado" (IPPDH, 2014, p. 140). Es necesario también reconocer que los desafíos nacionales son heterogéneos y que los retos resultan más acuciantes para los países con mayores brechas de bienestar, es decir, con un PIB per cápita más bajo, mayores tasas de dependencia demográfica, mercados de trabajo más informales, menor cobertura de la protección social, mayores niveles de pobreza y menor inversión social (Cecchini, Filgueira y Robles, 2014).

En segundo lugar, para avanzar hacia la construcción de sistemas universales de protección social, se requiere "trabajar coordinando acciones y estructuras, como forma 
de materializar la integralidad necesaria para lidiar con la complejidad no solo de la pobreza y de la desigualdad, sino también del bienestar objetivo y subjetivo" (Cunill-Grau, Repetto y Bronzo, 2015, p. 410). Realizar una visión integral de la protección social significa hacer cambios sustanciales en la gestión de las políticas públicas. Significa pasar de la implementación de múltiples programas sociales desarticulados a la elaboración de estrategias y políticas que tomen en cuenta las interrelaciones entre lo económico, lo medioambiental y lo social. Significa, asimismo, integrar y articular los servicios y las prestaciones de protección social para cumplir con todos los derechos de individuos, familias y comunidades, mediante la implementación de "ventanillas únicas" de protección social (Cecchini y Martínez, 2011), la promoción del "gobierno de proximidad", los procesos participativos y el fortalecimiento de los sistemas de información social (Cunill-Grau, Repetto y Bronzo, 2015).

Si bien la oferta sectorial de políticas y programas de protección social en los países de la región sigue caracterizándose por fuertes fronteras institucionales, las personas no se relacionan con las políticas sociales en forma fragmentada a la hora de enfrentar los problemas y riesgos, y de aprovechar las oportunidades existentes para el bienestar. Las personas consideran en un tiempo y espacio concreto el conjunto de prestaciones que están disponibles en los diferentes ámbitos (por ejemplo, salud, seguridad social o educación) y hacen uso de ellas también como un conjunto. Asimismo, debemos tener en cuenta que las decisiones no se restringen a la acción del Estado, sino que incluyen también los recursos disponibles en la familia y en el mercado (Cecchini, Filgueira, Martínez y Rossel, 2015). De allí la importancia de tener en cuenta las interacciones con otros actores -sean ellos prestadores privados $\mathrm{u}$ ONG- así como los incentivos y desincentivos que se pueden crear por estas interacciones.

En última instancia, adoptar el enfoque de derechos implica un cambio copernicano en las políticas y los programas de protección social, que pasan a ser parte de una política de Estado, y no solamente de gobierno, y que atienden a ciudadanos titulares de derechos, y no solo a personas con necesidades o riesgos. Más que de "beneficiarios" específicos, la protección social se ocupa, por tanto, de todos los ciudadanos, que son "destinatarios", "usuarios" o "participantes" de las políticas y programas (Cecchini y Rico, 2015). En lugar de beneficiarios pasivos, los ciudadanos pasan a ser protagonistas de su propio desarrollo (IPPDH, 2014) en el sentido republicano: empoderados, participativos, exigentes, demandantes e informados.

\section{REFERENCIAS}

Abramovich, V. (2006). Una aproximación al enfoque de derechos en las estrategias y políticas de desarrollo. En Revista de la CEPAL, 88. Santiago de Chile: Comisión Económica para América Latina y el Caribe (CEPAL), abril.

Artigas, C. (2005). Una mirada a la protección social desde los derechos humanos y otros contextos internacionales. En Serie Políticas Sociales, 110. Santiago de Chile, Comisión Económica para América Latina y el Caribe (CEPAL). 
Balakrishnan, R., Elson, D., Heintz, J. y Lusiani, N. (2011). Maximum Available Resources and Human Rights: Analytical Report. Rutgers: Centro para el Liderazgo Global de las Mujeres, The State University of New Jersey.

Barba, C. (2013). El nuevo paradigma de bienestar residual y deslocalizado: reforma de los regímenes de bienestar en la OCDE, América Latina y México. Tesis presentada para obtener el grado de Doctor en Ciencias Sociales por parte de la Universidad de Guadalajara y el Centro de Investigaciones y Estudios Superiores en Antropología Social.

Bobbio, N. (1996). Left and Right: The Significance of a Political Distinction. Chicago: The University of Chicago Press.

Boyer, R. (2015). Crecimiento, empleo y equidad: el nuevo papel del Estado. Neoestructuralismo y corrientes heterodoxas en América Latina y el Caribe a inicios del siglo xxi. En Bárcena, A. y Prado, A. (eds.). Neoestructuralismo y corrientes heterodoxas en América Latina y el Caribe a inicios del siglo XXI. Santiago de Chile: Comisión Económica para América Latina y el Caribe (CEPAL).

Cecchini, S., Filgueira, F., Martínez, R. y Rossel, C. (2015). Derechos y ciclo de vida: reordenando los instrumentos de protección social. En Instrumentos de protección social. Caminos latinoamericanos hacia la universalización. Santiago de Chile: Comisión Económica para América Latina y el Caribe (CEPAL).

Cecchini, S., Filgueira, F. y Robles, C. (2014). Sistemas de protección social en América Latina y el Caribe: una perspectiva comparada. En Serie Politicas sociales, 202. Santiago de Chile: Comisión Económica para América Latina y el Caribe (CEPAL).

Cecchini, S. y Martínez, R. (2011). Protección social inclusiva en América Latina: una mirada inte- gral, un enfoque de derechos. Santiago de Chile: Comisión Económica para América Latina y el Caribe (CEPAL).

Cecchini, S. y Rico, N. (2015). El enfoque de derechos en la protección social. En Cecchini, S., Filgueira, F., Martínez, R. y Rossel, C. (eds.). Instrumentos de protección social. Caminos latinoamericanos hacia la universalización. Santiago de Chile: Comisión Económica para América Latina y el Caribe (CEPAL).

Cecchini, S. y Vargas, L. H. (2015). Protección social, ajuste estructural y brechas de bienestar. Una perspectiva latinoamericana, presentado en el Primer Congreso Iberoamericano de Relaciones Laborales y Recursos Humanos. Los sistemas de relaciones laborales del siglo XIX a la actualidad. Sevilla: Sistemas de seguridad social.

CEPAL (Comisión Económica para América Latina y el Caribe) (2015a). Balance preliminar de las economias de América Latina y el Caribe 2015. Santiago de Chile: Naciones Unidas.

CEPAL (Comisión Económica para América Latina y el Caribe) (2015b). Panorama social de América Latina 2014. Santiago de Chile: Naciones Unidas.

CEPAL (Comisión Económica para América Latina y el Caribe) (2015c). Desarrollo social inclusivo. Una nueva generación de políticas para superar la pobreza y reducir la desigualdad en América Latina y el Caribe. Santiago de Chile: Naciones Unidas. CEPAL (Comisión Económica para América Latina y el Caribe) (2014). Pactos para la igualdad. Hacia un futuro sostenible. Santiago de Chile: Naciones Unidas.

CEPAL (Comisión Económica para América Latina y el Caribe) (2010). Panorama social de América Latina 2009. Santiago de Chile: Naciones Unidas.

CEPAL (Comisión Económica para América Latina y el Caribe) (2007). Cohesión social: inclusión y sen- 
tido de pertenencia en América Latina y el Caribe. Santiago de Chile: Naciones Unidas.

Cepal (Comisión Económica para América Latina y el Caribe) (2006). La protección social de cara al futuro: acceso, financiamiento y solidaridad. Santiago de Chile: Naciones Unidas.

Cunill-Grau, N., Repetto, F. y Bronzo, C. (2015). Coordinación intersectorial pro integralidad de las instituciones de protección social. En Cecchini, S., Filgueira, F., Martínez, R. y Rossel, C. (eds.). Instrumentos de protección social. Caminos latinoamericanos hacia la universalización. Santiago de Chile: Comisión Económica para América Latina y el Caribe (CEPAL).

Esping-Andersen, G. (1990). The Three Worlds of Welfare Capitalism. Princeton: Princeton University Press.

Esping-Andersen, G. (1999). Social Foundations of Postindustrial Economies. New York: Oxford University Press.

Esping-Andersen, G. (2002). Why We Need a New Welfare State. New York: Oxford University Press.

Evans, P. (2008). In Search of the $21^{\text {st }}$ Century Developmental State. Brighton: The Centre for Global Political Economy, University of Sussex.

Ferreira, F. y Robalino, D. (2010). Social protection in Latin America: Achievements and limitations. En Policy Research Working Paper, 5305. Washington, D.C.: Banco Mundial.

Gacitúa-Marió, E., Norton, A. y Georgieva, S. (2009).

Building Equality and Opportunity through Social Guarantees: New Approaches to Public Policy and the Realization of Rights. Washington, D.C.: Banco Mundial.

González de la Rocha, M. (2010). Pobreza, Progresa y Oportunidades: una mirada de relativo largo plazo. En Abrahão de Castro, J. y Modesto, L. (orgs.). Bolsa Família 2003-2010: avanços e de- safios (vol. 2). Brasilia: Instituto de Investigación Económica Aplicada (IPEA).

Habermas, J. (1998). The Inclusion of the Other: Studies in Political Theory. Cambridge мa: The мгт Press.

Hardy, C. (2014). Estratificación social en América Latina: retos de cohesión social. Santiago de Chile: LOM Ediciones.

Hevia de la Jara, F. y Gruenberg, C. (2010). Continuidades y reformas en el sistema de atención ciudadana del Programa de Desarrollo Humano Oportunidades. En Mexican Rural Development Research Reports, 11. Washington: Woodrow Wilson International Center for Scholars.

Holzmann, R. y Jorgensen, S. (1999). Social protection as social risk management: conceptual underpinnings for the social protection sector strategy paper. Journal of International Development, 11 (17).

Hopenhayn, M. (2001). Viejas y nuevas formas de la ciudadanía. En Revista de la CEPAL, 73. Santiago de Chile: Comisión Económica para América Latina y el Caribe (CEPAL).

IPPDH (Instituto de Políticas Públicas en Derechos Humanos del Mercosur) (2014). Ganar derechos. Lineamientos para la formulación de políticas públicas basadas en derechos. Serie Documentos de Trabajo, 2. Buenos Aires: IPPDH.

Lo Vuolo, R. (2009). Social exclusion policies and labour markets in Latin America. En Hujo, K. y Mcclanahan, S. (eds.). Financing Social Policy. Mobilizing Resources for Social Development. New York: Instituto de Investigaciones de las Naciones Unidas para el Desarrollo Social (UNRISD), Palgrave-Macmillan.

Marshall, T. H. (1950). Citizenship and Social Class and other Essays. Cambridge: Cambridge University Press. 
Martínez, R. y Fernández, A. (2009). El costo del hambre: impacto social y económico en el Estado Plurinacional de Bolivia, Ecuador, Paraguay y Perú. En Documentos de Proyecto, 260. Santiago de Chile: Comisión Económica para América Latina y el Caribe (CEPAL)/Programa Mundial de Alimentos (PMA).

Martínez, R. y Fernández, A. (2007). El costo del hambre. Impacto social y económico de la desnutrición infantil en Centroamérica y República Dominicana. En Documentos de Proyectos, 144. Santiago de Chile: Comisión Económica para América Latina y el Caribe (CEPAL)/Programa Mundial de Alimentos (PMA).

Martínez Franzoni, J. (2008). Domesticar la incertidumbre en América Latina: mercado laboral, politica social y familias. San José: Editorial de la Universidad de Costa Rica/UndP.

Mesa-Lago, C. (1977). Modelos de seguridad social en América Latina: estudio comparado. Buenos Aires, Argentina: Siap-Planteos.

Munro, L. (2008). Risks, needs and rights: compatible or contradictory bases for social protection. En Barrientos, A. y Hulme, D. (eds.). Social Protection for the Poor and Poorest. Concepts, Policies and Politics. Basingstoke: Palgrave-Macmillan.

Pierson, C. y Leimgruber, M. (2010). Intellectual roots. En Castles F. G., Leibried, S., Lewis, J., Obinger, H. y Pierson, C. The Oxford Handbook of the Welfare State, Oxford: Oxford University Press.

Ribe, H., Robalino, D. y Walker, I. (2010). De los derechos a la realidad. Una protección social eficaz para todos en América Latina y el Caribe. Washington D.C.: Banco Mundial.

Robles, C. y Mirosevic, V. (2013). Sistemas de protección social en América Latina y el Caribe:
Brasil. En Documentos de Proyecto, 532. Santiago de Chile: Comisión Económica para América Latina y el Caribe (CEPAL).

Segura-Ubiergo, A. (2012). The Political Economy of the Welfare State in Latin America. Cambridge: Cambridge University Press.

Sepúlveda, M. (2014). De la retórica a la práctica: el enfoque de derechos en la protección social en América Latina. En Serie Politicas Sociales, 189. Santiago de Chile: Comisión Económica para América Latina y el Caribe (CEPAL).

Serrano, C. y Raczynski, D. (2003). Derechos sociales básicos, superación de la pobreza y protección social ante la vulnerabilidad. Santiago de Chile: Asesorías para el Desarrollo http://www.asesoriasparaeldesarrollo.cl/secciones/areas_de_trabajo/ politicas_sociales.html.

Soares, S. (2012), Bolsa Família, its design, its impacts and possibilities for the future. En IPC-IG Working Paper, 89. Brasilia: Centro Internacional de Políticas para el Crecimiento Inclusivo (IPC-IG). Sønneland, M. (2014). El sistema de protección social en Noruega. En Cecchini, S. y Lavigne, M. (eds). Politicas públicas para la igualdad: hacia sistemas de protección social universal. Serie Seminarios y Conferencias, 78. Santiago de Chile: Comisión Económica para América Latina y el Caribe (CEPAL).

Uprimny, R. y Durán, J. (2014). Equidad y protección judicial del derecho a la salud en Colombia. En Serie Politicas Sociales, 197. Santiago de Chile: Comisión Económica para América Latina y el Caribe (CEPAL). 